\title{
Droplets and the Configurational Entropy Crisis for Random First Order Transitions
}

\author{
M. P. Eastwood and P. G. Wolynes \\ Department of Chemistry and Biochemistry, University of California, \\ 9500 Gilman Drive, La Jolla, CA 92093-0371, USA
}

(Dated: October 22, 2018)

\begin{abstract}
We consider the effect of droplet excitations in the random first order transition theory of glasses on the configurational entropy. The contribution of these excitations is estimated both at and above the ideal glass transition temperature. The temperature range where such excitations could conceivably modify or "round-out" an underlying glass transition temperature is estimated, and found to depend strongly on the surface tension between locally metastable phases in the supercooled liquid. For real structural glasses this temperature range is found to be very narrow, consistent with the quantitative success of the theory. For certain finite-range spin-glass models, however, the surface tension is estimated to be significantly lower leading to much stronger entropy renormalizations, thus providing an explanation for the lack of a strict thermodynamic glass transition in simulations of these models.
\end{abstract}

A number of models of disordered glassy systems such as random heteropolymers [1], $p$-spin glasses [2] and Potts spin glasses [3] in the mean field limit undergo a thermodynamic transition at which an appropriately defined configurational entropy vanishes. While supercooled liquids in the laboratory never reach such a transition before falling out of equilibrium, rather natural extrapolations of the experimentally measured thermodynamics also suggest that if a fluid could remain equilibrated upon slow cooling, an entropy crisis would occur at an ideal glass transition temperature $T_{\mathrm{K}}$ [4]. This temperature is remarkably near the temperature where, again a natural extrapolation of the experimental kinetic data suggests that relaxations become infinitely slow.

Over many decades, contemplating such an ideal glass transition has entered into discussions of glassy dynamics. The "random first order transition" (RFOT) exhibited by the mean-field models has become a central element of the modern theory of glasses 15, 6, 7, 8, 9, 10. Recently this theory has been shown to predict quantitatively from a microscopic starting point the dynamics of supercooled liquids in the laboratory [11, 12], including the relaxation time distribution and the length scale of dynamical heterogeneity 13. This theory also leads to a natural way to describe the anomalous thermodynamic and transport properties of low temperature glasses, when the theory is quantized 14]. Despite its venerable history 15 the idea of an underlying ideal glass transition related to an entropy crisis has also been greeted by much controversy since it requires extrapolation from extant data. Yet only one a priori objection to an entropy crisis has been crisply formulated: configurational entropy cannot vanish above absolute zero since a system with finite range forces can always support point defects at any finite temperature 16. The most naive incarnation of this objection, the existence of vacancies or interstitials, can easily be dismissed by practical persons. At the densities of glasses the concentration of such defects is miniscule. Reasonable estimates suggest they occur in 1 part in $10^{4}$ so they hardly contribute to the configurational entropy, which is typically $1 k_{B}$ per particle at the laboratory liquid-glass transition temperature, $T_{\mathrm{g}}$. More troubling is that the random first order transition theory itself requires the existence of excitations ("entropic droplets") which are localized and have finite energy cost. It is conceivable these excitations can smear out or modify the critical exponents of the transition predicted by mean-field theory. In this paper, we estimate what the droplet contribution to the entropy both below and above an ideal glass transition would be. This calculation parallels Fisher's droplet calculations on the essential singularities in the thermodynamics encountered near an ordinary first order phase transition 17 . The calculation shows how close to an ideal glass transition we must go to see these renormalization effects and quantifies where the notion of configurational entropy becomes ambiguous. In essence this calculation provides something like a "Ginzburg" criterion [18] describing the breakdown of mean-field theory for a random first order transition. The size of the strongly renormalized regime depends strongly on the effective surface tension between mean-field replica symmetry broken phases. The effective surface tension parameter is actually quite large for the liquid-glass transition because it depends on the Lindemann vibrational amplitude which is so small. Effectively there is, then, a small parameter in the theory which allows the mean-field estimates to prevail until very close to the transition (much as does the BCS theory of conventional superconductivity). The entropy crisis for liquids will be noticeably avoided only when the residual entropy is of the order $10^{-4} k_{B}$ and $\left(T-T_{K}\right) / T_{K}$ is approximately $10^{-4} k_{B} / \Delta c_{v}$ where $\Delta c_{v}$ is the mean-field heat capacity discontinuity at the transition. In contrast, the effective surface tension for a 10-state nearest neighbour lattice Potts glass is quite small. This small value is consistent with the avoidance of an entropy crisis in a recent simulation of this system [19]. Since the surface tension parameter also tunes the degree of slowing as the transition is approached understanding these effects may allow the design of simulations that can more effectively confront the theory.

We begin by very briefly summarizing the essential 
points of the RFOT theory of glasses, and then construct a simple partition function for the droplet excitations. After discussion of droplet effects at $T_{\mathrm{K}}$, we evaluate the temperature range over which such excitations will be significant for a structural glass as $T$ approaches $T_{\mathrm{K}}$ from above. Finally, we apply the results to several glassy systems with different surface tensions.

Below a temperature $T_{A}$, which corresponds to the dynamical glass transition predicted by mode-coupling theory [20], reconfiguration of a real supercooled liquid occurs by activated processes. According to the RFOT theory of glasses for $T_{\mathrm{K}}<T<T_{A}$ the driving force for reconfiguration of a locally frozen region is the configurational entropy, while there is an energetic cost due to the surface tension between two different locally metastable solutions. The free energy profile for reconfiguration of a region of radius $r$ is thus

$$
F(r)=4 \pi r^{2} \sigma(r)-\frac{4}{3} \pi r^{3} \rho T S_{c}
$$

where $S_{c}$ is the configurational entropy density per molecule, and $\rho$ is the number density. For the experimental comparison the measured $S_{c}$ was used, but the theory more formally would call for its mean-field value. Generalization of a renormalization group ( $R G)$ argument due to Villain [21] suggests that the surface tension is reduced from its bare value $\sigma_{0}$ (obtained between two typical metastable states) due to wetting of the interface by additional metastable states, according to $\sigma(r)=\sigma_{0}\left(r_{0} / r\right)^{\frac{1}{2}}$, with $r_{0}=\rho^{-\frac{1}{3}}$ a typical intermolecular distance 8. It is helpful to express the two key parameters (surface tension and configurational entropy) in terms of dimensionless parameters that are approximately constant as $T \rightarrow T_{\mathrm{K}}$. Specifically, the configurational entropy is assumed to vanish linearly, $S_{c}=\frac{T-T_{\mathrm{K}}}{T_{\mathrm{K}}} \frac{\Delta c_{v}}{k_{B}}$, where $\Delta c_{v}$ is the heat capacity jump per particle at the transition, and we define a reduced surface tension $A=4 \pi r_{0}^{2} \sigma_{0}\left(T_{\mathrm{K}}\right) / k_{B} T_{\mathrm{K}}$. In the region $T_{\mathrm{K}}<T<T_{A}$, we assume that $4 \pi r_{0}^{2} \sigma_{0}(T) / k_{B} T$ is slowly changing, and may be approximated by $A$. The reconfiguration barrier may then be written in Vogel-Fulcher form as $\beta \Delta F^{\ddagger}=D T_{\mathrm{K}} /\left(T-T_{\mathrm{K}}\right)$, with the fragility $D=\frac{3}{16 \pi} \frac{A^{2}}{\Delta c_{v} / k_{B}}$. The supercooled liquid is viewed as a mosaic of metastable regions of typical size $R / r_{0}=$ $\left(\frac{3 A}{4 \pi} \frac{k_{B}}{\Delta c_{v}}\right)^{\frac{2}{3}}\left(\frac{T_{\mathrm{K}}}{T-T_{\mathrm{K}}}\right)^{\frac{2}{3}}$, as follows from $F(R)=F(0)$. For structural glasses $\Delta c_{v} / k_{B}$ varies a great deal from substance to substance over a typical range of 0.2 to 5 . However, a density functional calculation [11] gives $A \approx 23$ for structural glasses approximately independently of molecular details since it depends only logarithmically on the Lindemann ratio of mean-square vibrational amplitude to near-neighbour spacing which characterizes the vibrations at $T_{\mathrm{g}}$.

Within each domain of the mosaic there should exist further droplet excitations with free energy profiles also given by eq. (11), as shown schematically in fig. 1. Indeed, since these droplets may also contain even

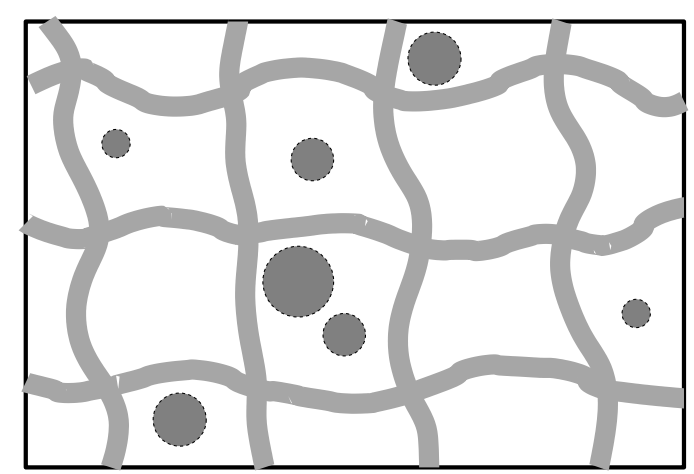

FIG. 1: A schematic of the structure of a supercooled liquid near $T_{\mathrm{K}}$, showing droplet excitations within the mosaic cells.

smaller droplets, this suggests that like the surface tension the configurational entropy should be determined by $\mathrm{RG}$ equations. In this paper, we estimate the region where such a renormalization should be significant and leave the full RG calculation for future work, although we note that in the case of the random field Ising model, the presence of droplets within droplets is not sufficient to raise the lower critical dimension from $d=2$ to $d=3$ [22, 23]. The partition function for droplet formation within a single mosaic cell is $Z=\sum_{\left\{n_{j}\right\}} \exp \left(S_{\text {comb }}\left(\left\{n_{j}\right\}\right)-\beta \sum_{i} n_{i} F\left(r_{i}\right)\right)$, where the free energy cost of forming a droplet of radius $r_{i}$ about a given position is given by eq. (II), and $S_{\text {comb }}$ is an entropy associated with the different positions the droplets may take up within the domain. Assuming that there is approximately one distinct droplet position for each molecule that a droplet may be centered on, gives $\frac{1}{n_{i} !}\left(\rho V_{e f f}\right)^{n_{i}}$ states of $n_{i}$ identical independent droplets. The effective volume within a domain in which a droplet may move, $V_{\text {eff }}=\frac{4}{3} \pi R_{\text {eff }}^{3}$, is taken to be smaller than the domain volume due to the presence of the domain walls of finite thickness. While strictly speaking the RG wetting argument suggests $R_{\text {eff }} \propto R$ [8], with essentially no effect on the results we take $R_{\text {eff }}=R-r_{0}$. Excluded volume effects have been entirely ignored, even between identical droplets, as has the reduction in the volume available to position larger droplets due to the restriction that the entire droplet must lie within the domain walls (this latter effect may be straightforwardly included, but is not significant for parameters where ignoring the excluded volume is a good approximation). Under these approximations the entropy $S_{c o m b}$ reduces to $S_{\text {comb }}=\sum_{i}\left\{n_{i} \ln \left[\rho V_{\text {eff }}\right]-\ln n_{i} !\right\}$, and the partition function may easily be evaluated giving a free energy density of independent droplets $F_{\text {drop }}=-\left(k_{B} T / \rho V\right) \ln Z=$ $-k T\left(V_{e f f} / V\right) \sum_{i} e^{-\beta F_{i}}$. Droplets may be distinguished by the number of molecules they contain, so what is meant by the sum over droplet types $i$ in eq. (2) is $i=1,2, \ldots, i_{\max }$, where $i_{\max }$ is the maximum size of a droplet that can fit inside the domain $i_{\max } \leq \rho V_{\text {eff }}$; alternatively — and no more approximately — we may 
make the replacement $\sum_{i=1}^{i_{\max }} \rightarrow 4 \pi \rho \int_{r_{s}}^{R_{\text {eff }}} r^{2} d r$, with $r_{s}=\left(\frac{3}{4 \pi}\right)^{\frac{1}{3}} r_{0} \approx 0.62 r_{0}$. The corresponding droplet entropy density (which follows from differentiation with $S_{c}$ and $\sigma_{0}$ fixed) is

$$
S_{\text {drop }} / k_{B}=4 \pi \rho \frac{V_{\text {eff }}}{V} \int r^{2}\left(1+A\left(r / r_{0}\right)^{\frac{3}{2}}\right) e^{-\beta F(r)} d r .
$$

At $T_{\mathrm{K}}$ the droplet entropy density $S_{d r o p}\left(T_{\mathrm{K}}\right) / k_{B}=$ $(8 \pi / 3)\left[\tilde{r}_{s}^{3}+3 \tilde{r}_{s}^{\frac{3}{2}} / A+3 / A^{2}\right] \exp \left(-A \tilde{r}_{s}^{\frac{3}{2}}\right)$, where $\tilde{r}_{s}=$ $r_{s} / r_{0}$. For a structural glass, with the values given above, $S_{\text {drop }}\left(T_{\mathrm{K}}\right) / k_{B} \approx 3 \times 10^{-5}$. For structural glasses, the contribution to the droplet entropy comes overwhelmingly from the smallest droplets and is thus a defect-like entropy. Unfortunately, on the short length scales of such droplets (perhaps involving only a single molecule) the calculation of the energetic cost in terms of a surface tension becomes less accurate and the details of the particular molecules may become relatively more important. The effect of the uncertainty in the energetic cost of forming small droplets on the droplet entropy may be allowed for by permitting $\tilde{r}_{s}$ to vary in the range 0.4 to 1.0 , which corresponds to the energy of the smallest droplets being a factor of two smaller or larger than estimated using $\tilde{r}_{s}=0.62$. Even allowing for this uncertainty the resultant droplet entropy at $T_{\mathrm{K}}$ remains tiny, in the range $1 \times 10^{-9}$ to $3 \times 10^{-3} k_{B}$.

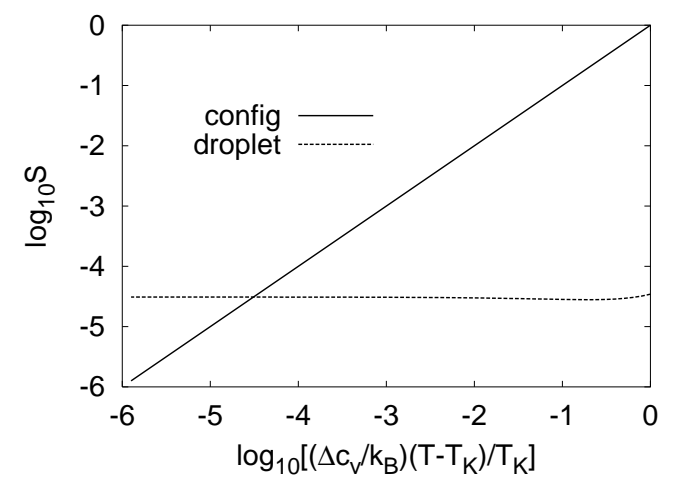

FIG. 2: The configurational entropy and the droplet correction to the entropy for a structural glass as a function of temperature in the temperature range $\left(T-T_{\mathrm{K}}\right) / T_{\mathrm{K}}=$ $10^{-6} k_{B} / \Delta c_{v}$ to $k_{B} / \Delta c_{v}$.

We now consider $T>T_{\mathrm{K}}$ and estimate the crossover temperature, $T_{\mathrm{x}}$, where the droplet entropy and configurational entropy become comparable. For $T_{\mathrm{K}}<T<T_{\mathrm{x}}$ droplet effects may modify the character of the random first order transition. As $S_{d r o p}\left(T_{\mathrm{K}}\right)$ is finite and small, it is clear that $T_{\mathrm{x}}>T_{\mathrm{K}}$ exists and since, as fig. 2 shows, $S_{\text {drop }}(T)$ is not rapidly varying as $T$ approaches $T_{\mathrm{K}}, T_{\mathrm{x}}$ may be estimated from from $S_{d r o p}\left(T_{\mathrm{K}}\right)=S_{c}(T)$. For large values of the reduced surface tension and $\tilde{r}_{s}=$ 0.62 this leads to $\log _{10}\left[\left(\Delta c_{v} / k_{B}\right) \times\left(T_{\mathrm{x}}-T_{\mathrm{K}}\right) / T_{\mathrm{K}}\right]=$ $-0.21 A+0.3$. For structural glasses this suggests $\left(T_{\mathrm{x}}-\right.$ $\left.T_{\mathrm{K}}\right) / T_{\mathrm{K}}$ lies in the range $1.5 \times 10^{-4}$ (for strong liquids) to $6 \times 10^{-6}$ (for fragile liquids). Such cross-over temperatures lie well below those accessible to equilibrium measurements, since the $\alpha$ relaxation times at $T_{\mathrm{x}}$ will be of the order $\tau_{0} \exp \left(10^{6}\right)$, effectively infinite. We show in fig. 3 the cross-over temperature as a function of $\Delta c_{v}$ over a range of $\Delta c_{v}$ appropriate to a structural glass. The widening of the renormalized region with decreasing $\Delta c_{v}$ (increasing fragility) is clear. The conclusion that $T_{\mathrm{x}}$ is inaccessible to direct experimental study for structural glasses appears robust to the uncertainties in the energy cost calculation: even taking $\tilde{r}_{s}=0.38$ (corresponding to an energy cost of half the natural estimate) leads to $\left(\Delta c_{v} / k_{B}\right) \times\left(T_{\mathrm{x}}-T_{\mathrm{K}}\right) / T_{\mathrm{K}}=2 \times 10^{-3}$ while $\left(\Delta c_{v} / k_{B}\right) \times\left(T_{\mathrm{g}}-T_{\mathrm{K}}\right) / T_{\mathrm{K}} \approx 1$.

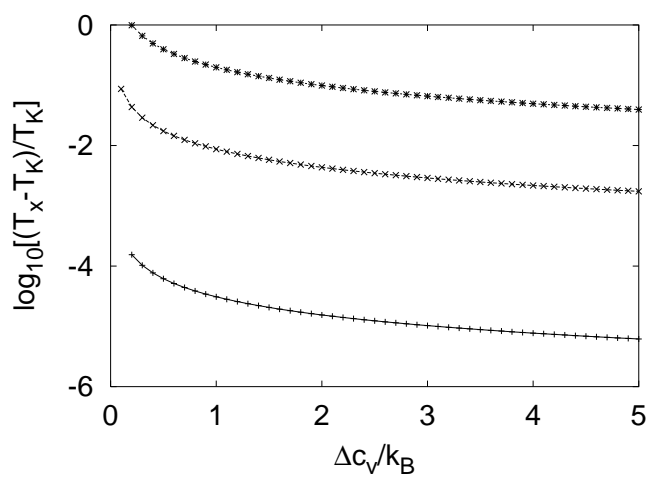

FIG. 3: $\log _{10}\left[\left(T_{\mathrm{x}}-T_{\mathrm{K}}\right) / T_{\mathrm{K}}\right]$, where $T_{\mathrm{x}}$ is the crossover temperature as a function of the heat capacity jump, for $A=23$ (bottom; appropriate for a structural glass), $A=12$ (middle; as may be appropriate for a protein-like random heteropolymer), and $A=6$ (top; possibly appropriate for some spin models as discussed in the text).

While structural glasses all have $A \approx 23$ other glassy systems are expected to have different bare surface tensions. The width of the renormalized region depends strongly on $A$. Figure 4 gives the full dependence of the width of the renormalized region on the bare surface tension parameter $A$. Also shown is the position of the laboratory glass transition temperature $T_{g}$, defined in terms of the experimental and microscopic times via $\tau_{g} / \tau_{0}=\exp \left(D T_{\mathrm{K}} /\left(T_{g}-T_{\mathrm{K}}\right)\right)$ with both $\tau_{g} / \tau_{0}=10^{17}$ (applicable to experiments on structural glasses with relaxations in the hours range) and $\tau_{g} / \tau_{0}=10^{8}$ (typical of a computer simulation of a spin glass). For large values of the surface tension $(A>15) T_{\mathrm{x}}$ is found to lie significantly below $T_{g}$ and the additional droplet entropy is small at any temperature where equilibration can be achieved in practice. For surface tensions in the range $5<A<10$, on the other hand, the values of $T_{\mathrm{x}}$ and $T_{g}$ will be more comparable and the effects of droplet excitations should become apparent. Depending on the details of the model and the timescale accessible to the experimenter as $A$ is decreased from 10 to 5 , at some point $T_{\mathrm{x}}$ and $T_{g}$ will cross and the effect of the droplet renormalization may be large. We note that by $A=6$ the 
independent droplet approximation breaks down since $\sim 30 \%$ of the volume of domains is predicted to be filled with droplet excitations at $T_{\mathrm{x}}$. Nevertheless, this does not change the conclusion that droplets are significant at this surface tension.

To use the droplet entropy renormalization criterion we need to know $A$ for systems with finite-ranged interactions that undergo RFOTs in the mean-field limit. Unfortunately, for protein, Potts and $p$-spin models accurate calculations of $A$ are somewhat involved. Therefore, here we content ourselves to estimate the value of $A$ for various models using rough calculations, that should be sufficiently accurate to determine whether or not droplet excitations are likely to play an important role in the entropy. We first make an estimate for polymeric, and then some spin-glass models.

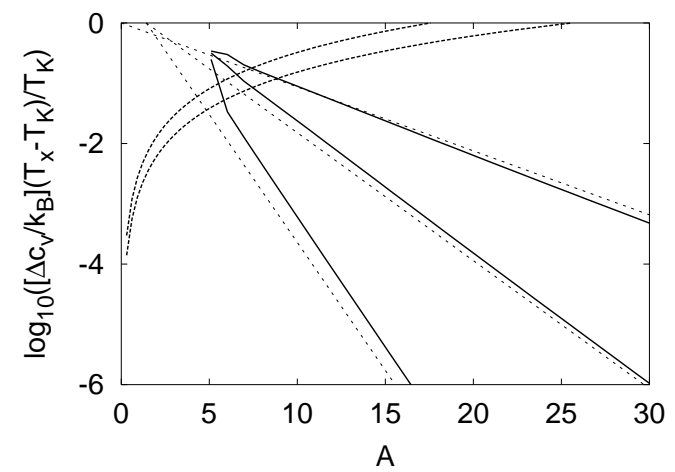

FIG. 4: Logarithm of the width of the renormalized region as a function of the dimensionless surface tension parameter. The three solid curves correspond (from top to bottom) to $\tilde{r}_{s}=0.38,0.62$ and 0.98 , with $T_{\mathrm{x}}$ calculated from $S_{c}\left(T_{\mathrm{x}}\right)=$ $S_{d r o p}\left(T_{\mathrm{x}}\right)$ and eq. (2). The three dotted lines are the asymptotes $\ln \left[\left(\Delta c_{v} / k_{B}\right) \times\left(T_{\mathrm{x}}-T_{\mathrm{K}}\right) / T_{\mathrm{K}}\right]=-\tilde{r}_{s}^{\frac{3}{2}} A+\ln \left[8 \pi \tilde{r}_{s}^{3} / 3\right]$. The two dashed lines give the laboratory glass transition temperature as $\log _{10}\left[\left(\Delta c_{v} / k_{B}\right) \times\left(T_{\mathrm{g}}-T_{\mathrm{K}}\right) / T_{\mathrm{K}}\right]$ for waiting times appropriate to experiments on structural glasses (lower) and computer simulations on spin-glasses (upper).

The surface tension for a random heteropolymer may be estimated using the argument of Takada and Wolynes [24], or alternatively by approximating the surface tension between two neighboring misfolded regions of a random heteropolymer with the surface tension of a sharp interface between folded and unfolded regions of a protein. The latter quantity is a key quantity in the capillarity model of protein folding [25, 26], which in its simplest form assumes folding occurs by nucleation with a sharp interface between completely folded and completely unfolded regions. At the folding temperature, $T_{f}$, the capillarity model predicts a free energy barrier of $\Delta F / k_{B} T_{f}=\frac{4}{27}\left(\frac{3}{4 \pi}\right)^{\frac{2}{3}} A N^{\frac{2}{3}}$. A knowledge of the variation of folding rates at $T_{f}$ with protein size will thus give an estimate of the reduced surface tension. Such a study has been performed by Koga and Takada 27, who find for simulations of model proteins a folding rate that varies as $\log _{10} k_{f} \approx-0.5 c N^{\frac{2}{3}}$, with $c$ a roughly $N$ independent constant that depends on protein topology called relative contact order. $c$ measures how frequently the chain returns to itself. The same relation holds for real proteins but folding times vary over more than four more orders of magnitude and the coefficient is large, $\log _{10} k_{f} \approx-2.3 c N^{\frac{2}{3}}$. $c$ is in the range 0.08 to 0.2 giving a range of values for $A$ from 7 to 19 for a real proteins in the laboratory, suggesting that droplet renormalization is not significant for the majority of cases. For the simulation models, on the other hand, the calculation gives $A$ in the range 1.6 to 4 indicating that droplet renormalization is likely to be very significant for off-lattice models of random heteropolymers.

TABLE I: Estimates of the reduced surface tension parameter $A$ for some models studied by simulation. $A_{s p}$ is the sharp interface approximation, while $A_{\xi}$ and $A_{\tau}$ are the results of extracting $A$ from the simulation data (using the RFOT theory) on the correlation length and relaxation times respectively.

\begin{tabular}{|c|c|c|c|c|}
\hline model & $k$ & $A_{s p}$ & $A_{\xi}$ & $A_{\tau}$ \\
\hline$p=4 M=3 \quad 28,29$ & $1 / 6$ & 10 & 4.8 & - \\
\hline$p=4$ plaquette $[30$ & $1 / 3$ & 10 & - & 3.3 \\
\hline$q=10$ Potts 19 & $1 / 6$ & 8 & - & - \\
\hline
\end{tabular}

Computer simulations have been performed on a few spin-glass systems that exhibit a one-step RSB transition in the mean-field limit including $p=4$ spin models on different lattices [29, 30, 31 and $q=10$ state Potts glass [19]. Unlike the $p=4$ spin models the Potts model did not display the characteristic signs of a glass transition. An estimate for $A$ for these systems based on the sharp short-range interface argument is clouded by the nearness of the mean-field $T_{A}$ and $T_{\mathrm{K}}$ for these models. If we nevertheless assume a sharp planar interface, and take the free energy cost of forming the interface to be approximately one half of the energetic cost of randomizing the interactions across the interface, we would find $r_{0}^{2} \sigma_{0}=k\left|E_{k}\right|$ (or $A=4 \pi k\left|E_{\mathrm{K}}\right| / T_{\mathrm{K}}$ ) with $k$ the fraction of interactions per site that are broken in forming the interface and $E_{\mathrm{K}}$ the energy per site at $T_{\mathrm{K}}$. The resulting estimates for $A$ using $E_{\mathrm{K}}$ and $T_{\mathrm{K}}$ from simulations (or from mean-field theory in the case of the Potts model) are shown in the table. Taken at face value, these suggest that the $p=4$ spin models have a surface tension only just sufficient to avoid substantial corrections to mean-field theory, while there may be significant renormalization for the 10-state Potts model. Alternatively an estimate of the surface tension can be obtained using the observed growth in correlation length 29] and relaxation times 30 which indeed have the functional forms predicted by RFOT theory. In doing this it is necessary to assume the theoretical results apply outside the region $T_{\mathrm{K}}<T \ll T_{A}$. These estimates, also given in the table are much lower. These low values are consistent with the observation that $T_{A}$ (where surface tension vanishes in finite ranged models) lies only slightly above $T_{\mathrm{K}}$ for these models in the mean-field limit; in particular 
$T_{A} / T_{\mathrm{K}}=1.009$ for the 10 -state Potts glass 32]. We conclude that the apparent lack of a glass transition in the 10-state Potts model is due to a low effective microscale surface tension. Since $\left|E_{\mathrm{K}}\right| / T_{\mathrm{K}}=2 S_{0}$ (in a random energy model, with $S_{0}$ the total entropy per site) it can be anticipated that $A$ will increase approximately logarithmically with the number of states in the Potts model but proportional to $p$ in a $p$-spin model [32]. Achieving spin systems with a surface tension similar to that in a structural glass would require a $p \approx 20(M=p-1)$ spin model, but a $q=10^{3}$ (or even higher) state Potts glass. Unfortunately spin models that are relatively easily simulated appear to have substantial corrections to the current form of RFOT theory while real structural glasses and proteinlike random heteropolymers have much smaller corrections.

\section{Acknowledgments}

This work was supported by NIH grant 5 R01 GM44557.
[1] E. I. Shakhnovich and A. M. Gutin, Eur. Lett. 8, 327 (1989).

[2] E. Gardner, Nuc. Phys. 257B, 747 (1985).

[3] D. J. Gross, I. Kanter, and H. Sompolinsky, Phys. Rev. Lett. 55, 304 (1985).

[4] W. Kauzmann, Chem. Rev. 43, 219 (1948).

[5] T. R. Kirkpatrick and P. G. Wolynes, Phys. Rev. A 35, 3072 (1987).

[6] T. R. Kirkpatrick and P. G. Wolynes, Phys. Rev. B 36, 8552 (1987).

[7] T. R. Kirkpatrick and D. Thirumalai, Phys. Rev. Lett. 58, 2091 (1987).

[8] T. R. Kirkpatrick, D. Thirumalai, and P. G. Wolynes, Phys. Rev. A 40, 1045 (1989).

[9] M. Mezard and G. Parisi, Phys. Rev. Lett. 82, 747 (1999).

[10] M. Mezard and G. Parisi, J. Phys. Condens. Matter 12, 6655 (2000).

[11] X. Xia and P. G. Wolynes, Proc. Natl. Acad. Sci. USA 97, 2990 (2000).

[12] X. Xia and P. G. Wolynes, J. Phys. Chem. B 105, 6570 (2001).

[13] X. Xia and P. G. Wolynes, Phys. Rev. Lett. 86, 5526 (2001).

[14] V. Lubchenko and P. G. Wolynes, Phys. Rev. Lett. 87, 195901 (2001).

[15] G. Adam and J. H. Gibbs, J. Chem. Phys. 43, 139 (1965).
[16] F. H. Stillinger, J. Chem. Phys. 88, 7818 (1988).

[17] M. E. Fisher, Physics 3, 255 (1967).

[18] V. L. Ginzburg, Sov. Phys. Sol. State 2, 1824 (1960).

[19] C. Brangian, W. Kob, and K. Binder, Preprint condmat/0202232.

[20] W. Götze, Aspects of structural glass transitions, in Liquids, freezing and glass transition, volume 1, pages 287-503, Elsevier Science Publishers B. V. 1991.

[21] J. Villain, J. Physique 46, 1843 (1985).

[22] J. Z. Imbrie, Phys. Rev. Lett. 53, 1747 (1984).

[23] J. Z. Imbrie, Commun. Math. Phys. 98, 145 (1985).

[24] S. Takada and P. G. Wolynes, J. Chem. Phys. 107, 9585 (1997).

[25] P. G. Wolynes, Proc. Natl. Acad. Sci. USA 94, 6170 (1997).

[26] A. V. Finkelstein and A. Y. Badretinov, Fold. Des. 2, 115 (1997).

[27] N. Koga and S. Takada, J. Mol. Biol. 313, 171 (2001).

[28] M. Campellone, G. Parisi, and P. Ranieri, Phys. Rev. B 59, 1036 (1999).

[29] S. Franz and G. Parisi, Eur. Phys. J. B 8, 417 (1999).

[30] D. Alvarez, S. Franz, and F. Ritort, Phys. Rev. B 54, 9756 (1996).

[31] H. Rieger, Physica A 184, 279 (1992).

[32] E. D. Santis, G. Parisi, and F. Ritort, J. Phys. A: Math. Gen. 28, 3025 (1995). 